\title{
Pagdalumat sa kaalaman ng mga mag-aaral ng senior high school sa sulating pananaliksik
}

\author{
Ojos, Maria Kathrina H. $\square$ \\ Cadandanan National High School, Bulan, Sorsogon, Philippines (mariakathrina.ojos@deped.gov.ph) \\ Deyto, Nilda H. \\ Sorsogon State University, Philippines (deytonilda@gmail.com) \\ Janer, Susan S. \\ Sorsogon State University, Philippines (sihjaner@sorsu.edu.ph)
}

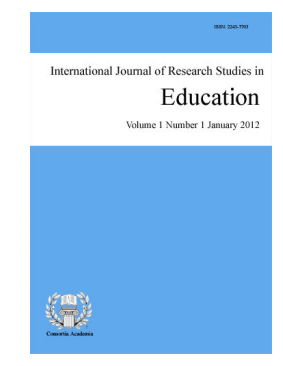

ISSN: 2243-7703 Online ISSN: 2243-7711

OPEN ACCESS

Received: 4 January $2022 \quad$ Revised: 9 January $2022 \quad$ Accepted: 11 January 2022

\section{Abstract}

This study aimed to determine the knowledge of Senior High School of Sorsogon National High School in research writing for school year 2021-22. The respondents of the study were composed of 40 students from grade 12 class, 20 students from General Academic Tract and 20 from TLE \& TVL Tract. Descriptive survey research design was used to gather the needed data. To determine the level of knowledge in research writing the researchers provide questionnaire to be answered by the respondents. The test question deals with the content, construction and mechanics in writing a research paper. Survey checklist was used to determine the factors that can affect the skills of the students in research writing. The data gathered were analyzed and interpreted using mean percentage, T-test, Scheffes Test, Frequency count and ranking. Results of the study revealed the following: the level of knowledge of Senior High School students on content, construction and mechanics are below $75 \%$ or did not meet expectation. Only the Academic tract passed the rating in mechanics which gather the $75.5 \%$ or fairly satisfactory. They have significant difference in content, construction and mechanics. Analysis revealed that General Academic tract got the highest rating compare to TLE-TVL. Based on the result the two strands agreed that constructing research title is the number one factor affecting the skills of the Senior High School in writing a research paper.

Keywords: knowledge, senior high school, research writing, general academic tract, TVL \& TLE 


\section{Pagdalumat sa kaalaman ng mga mag-aaral ng senior high school sa sulating pananaliksik}

\section{Introduksyon}

Malaki ang ambag ng pananaliksik sa pagbabago ng ating buhay, mula sa pag-unlad ng teknolohiya, pagdebelop ng mga bagong gamot at aparatu sa larangan ng siyensya, pagpapakilala ng iba't ibang inobasyon sa pagkain at istilo sa fashion, at maging sa larangan ng edukasyon.

Maraming mga pagbabago sa kurikulum ng edukasyon ang nangyari upang kayaning makipagsabayan ng mga magsisipagtapos sa global na larangan. Isa sa naging kontrobersyal na pagbabago sa edukasyon ay ang pagpapatupad ng K+12. Noong nakaraang 2012 nilagdaan ng dating pangulong Benigno Aquino Jr. ang Repulic Act No. 10533 o mas kilalang K+12 Law. Ayon sa dating Pang. Aquino sa pagsasabatas ng K+12, hindi lang nagdaragdag ng dalawang taon para sa higit pang pagsasanay ng mga mag-aaral; tinitiyak din na nabibigyang-lakas ang susunod na henerasyon na makiambag sa pagpapalago ng ekonomiya at lipunan. Bahagi ng kurikulum na ito ang pagdadagdag ng dalawang taon sa high school. Ilan sa mga asignaturang kabilang sa core subjects sa grade 11 na dapat mapag-aralan ng mga senior high school ay ang asignaturang Pagbasa at Pagsusuri ng Iba't ibang Teksto Tungo sa Pananaliksik (SHS Pagbasa at Pagsusuri ng Iba't ibang Teksto Tungo sa Pananaliksik CG 2017). Inaasahan sa asignaturang ito na makabubuo ng isang maikling pananaliksik na napapanahon ang paksa ang mga mag-aaral (F11EP-IVij-38).

Batay sa pag-aaral ni Balona, (2018) na nakatuon sa pagsulat ng mga personal na sanaysay,74 mula sa 100 kalahok na mag-aaral ng Bulan Cluster ang nakakuha ng bagsak na marka sa pagsulat ng sanaysay, 21 naman ang nakakuha ng fairly satisfactory at 5 lamang ang nakapasa. Natuklasan din sa pananaliksik na ito na ang mga mag-aaral ay nahihirapang bumaybay, gumamit ng tamang bantas at ipahayag ang sarili. Maaaring isipin na isa sa mga dahilan kung bakit mababa ang nakapasa sa pagsulat ng sanaysay ay dahil sa hindi na gaanong napagtutuunan ng pansin ng mga mag-aaral ang pagsulat.

Sa naging resulta ng pag-aaral maaaring isiping magiging balakid ang mga kahinaan nabanggit sa pagsulat ng mga mag-aaral ng sulating pananaliksik sa grade 11. Binigyang-diin ni Harry Shaw, (Vinuya,1997) na ang pananaliksik ay isang mahabang komposisyon na ibinibigay sa mga kurso sa kolehiyo at nangangailangan ng pagbasa ng maraming pinagkukunan ng impormasyon. Ang pananaliksik ay hindi lamang report kundi isang masusing imbestigasyon ng isang paksa para makagawa ng paglalahad, interpretasyon, at konklusyon mula sa mga babasahing ginamit ayon sa kung ano ang natuklasan.

Batay sa naging obserbasyon ng mga mananaliksik ang pagsulat ng sulating pananaliksik ay hindi nabigyan ng tuon sa junior high school. Kaya, maaaring isipin na magkakaroon ng kabiglaan sa parte ng mga mag-aaral dahil nasanay silang ang pagsulat ay madalas pagpapahayag lamang ng damdamin. Sa kanilang pagpasok sa grade 11 sila ay inaasahang makapagsulat ng isang pananaliksik tungkol sa napapanahong paksain.

Nilalayon ng pananaliksik na ito na masukat ang antas ng kaalaman ng mga mag-aaral sa senior high school mula sa iba't ibang track sa sulating pananaliksik. Nais suriin sa pananaliksik na ito ang kaalaman ng mag-aaral sa nilalaman, pagkakabuo, at mekaniks. Gayundin, aalamanin din ang mga salik na nakakaapekto sa kanilang pagsulat. Inaasahan ng mananaliksik na makabubuo ng mungkahing gawain upang matugunan ang mga kahinaan na natuklasan sa pag-aaral.

\subsection{Paglalahad Ng Suliranin}

Layunin sa pananaliksik na ito na matukoy ang kaalaman ng senior high school sa sulating pananaliksik sa Mataas na Paaralan ng Lungsod ng Sorsogon taong panuruan 2021 - 2022. Sasagutin ng sumusunod na 
Pagdalumat sa kaalaman ng mga mag-aaral ng senior high school sa sulating pananaliksik

katanungan. (1) Ano ang antas ng kaalaman ng mag-aaral sa Grade 12 sa sulating pananaliksik ayon sa: Nilalaman, Pagkakabuo, at Mekaniks? (2) Ano ang makabuluhang pagkakaiba ng antas ng kaalaman ng mga mag-aaral sa iba't ibang track ayon sa mga nabanggit na baryabols? At (3) Ano ang mga salik na nakakaapekto sa kakayahan ng mga mag-aaral sa pagsulat ng pananaliksik ayon sa mga nabanggit na baryabol?

\subsection{Pamamaraang ginamit}

Deskriptibong sarbey ang ginamit sa paglikom ng datos o impormasyon. Sa pagsukat sa antas na kaalaman ng 40 na kalahok sa sulating pananaliksik isang pagsusulit ang inihanda ng mananaliksik na sinagutan ng 20 na kalahok na kumukuha ng General Academic Strand (GAS) sa Academic Track at 20 kalahok naman mula sa Cookery sa TLE \& TVL Track. Ang pagsusulit ay sinuri at dumaan sa balidasyon, ito ay nakatuon sa kaalaman tungkol sa sulating pananaliksik: nilalaman, pagkakabuo, at mekaniks. Isang tseklist naman ang sinagutan ng mga kalahok upang malaman ang mga salik na nakaaapekto sa kakayahan ng mga mag-aaral sa sulating pananaliksik. Ang mga nalikom na datos ay sinuri at binigyang interpretasyon sa pamamagitan ng nararapat na istadistika - mean percentage, T-test, Scheffe's test, frequency count at pagraranggo.

\section{Mga Natuklasan}

Antas ng kaalaman ng mga mag-aaral sa Grade 12 ng iba't ibang track sa sulating pananaliksik ayon sa nilalaman, pagkakabuo, at mekaniks.

\subsection{Antas ng kaalaman ayon sa nilalaman ng sulating pananaliksik}

Sa suliranin ng pananaliksik na ito, tinukoy ang antas ng kaalaman ng mga mag-aaral sa Grade 12 ng iba't ibang track sa sulating pananaliksik ayon sa iba't ibang baryabol. Upang matukoy ang antas ng kaalaman ng mga mag-aaral sa nilalaman ng sulating pananaliksik, ang mga sumusunod na kasanayang pampagkatuto ang pinagbasehan ng mga tanong sa sarbey: (1) Nasusuri ang mga katangian ng mabuting pananaliksik sa pagpili ng paksa sa pananaliksik, at (2) natutukoy ang tamang paghahanguan ng datos na may kaugnayan sa pag-aaral.

\section{Talahanayan 1A}

Antas ng kaalaman ng mga mag-aaral sa Grade 12 ng iba't ibang track sa sulating pananaliksik ayon sa nilalaman

\begin{tabular}{lcc}
\hline \multicolumn{1}{c}{ Track } & Rating $(\%)$ & Antas ng Kaalaman \\
\hline Academic Track & 72.5 & Did not meet expectation \\
TLE and TVL & 69.8 & Did not meet expectation \\
Kabuuang rating & 71.1 & Did not meet expectation \\
\hline
\end{tabular}

Batay sa datos na nasa Talahanayan 1A, ang Academic Track ay nakakuha ng rating na 72.5\%, TLE and TVL Track ay $69.8 \%$. Ang parehong track ay nasa did not meet expectation at hindi umabot sa inaasahang pasadong rating na $75 \%$ o fairly satisfactory.

Nangangahulugan itong kulang ang kaalaman ng mga mag-aaral sa senior high school sa sulating pananaliksik ayon sa nilalaman. Ang naging tuon ng pagsusulit sa nilalaman ay ang sa pagtukoy ng suliranin o paksa sa pananaliksik, pag-alam sa mga maaaring baryabol, at mga mapagkukunan o paghahanguan ng mga paksa sa pananaliksik.

Magiging balakid ang kakulangan ng kaalaman ng mga mag-aaral sa nilalaman sapagkat ang pagbuo ng isang pananaliksik ay nagsisimula sa pagpili ng paksa o pagkakaroon ng malinaw na konsepto sa paksa ng isang pananaliksik. Ito din ang magiging batayan sa pagkuha ng mga ilalagay na datos, at magsisilbing gabay upang magkaroon ng pokus ang isang pananaliksik, kaya mahalagang pagplanuhan ang pagpili ng paksa.

Marami ang mga pagsubok na kinahaharap ng mga mag-aaral sa pagbuo ng isang saliksik. Upang makabuo 
ng isang wasto at makabuluhang saliksik, ang isang mag-aaral ay dapat na mayroong malinaw na kaalaman sa paksa na nais niyang pag-aralan. Ang pagpili ng paksa ay isa sa mga pinakamahalagang hakbang sa kabuuang proseso ng pananaliksik (Alvesson et al., 2013). Ayon kina Anson at Smith (2004), ang mga mag-aaral ay nahihirapang magdesisyon kung ano ba ang mga paksa ng pananaliksik na magagawa at iilan lang ang may malinaw na pokus sa kung ano ang dapat nilang gawin sa saliksik. Ang kawalan ng malinaw na pokus ang siyang nagiging malaking balakid upang makapagsimula ang mag-aaral sa pananaliksik.

\subsection{Antas ng kaalaman ayon sa pagkakabuo ng sulating pananaliksik}

Upang matukoy naman ang antas ng kaalaman ng mga mag-aaral ayon sa pagkakabuo ng sulating pananaliksik, ang mga tanong sa survey ay inilapat sa mga sumusunod na kasanayang pampagkatuto: (1) natutukoy ang tamang pagkakasunod-sunod ng bahagi ng pananaliksik, at (2) natutukoy ang mga pamamaraan sa pangangalap ng datos. Layunin nito na malaman ang antas ng kaalaman ng mga mag-aaral sa tamang pagkakasunod-sunod ng mga bahagi ng isang pananaliksik.

\section{Talahanayan 1B}

Antas ng kaalaman ng mga mag-aaral sa Grade 12 ng iba't ibang track sa sulating pananaliksik ayon sa pagkakabuo

\begin{tabular}{lcc}
\hline \multicolumn{1}{c}{ Track } & Rating $(\%)$ & Antas ng Kaalaman \\
\hline Academic Track & 74.0 & Did not meet expectation \\
TLE and TVL & 69.3 & Did not meet expectation \\
Kabuuang rating & 71.65 & Did not meet expectation \\
\hline
\end{tabular}

Sa talahanayan 1B ay makikita ang resulta ng pagsusulit ng mga mag-aaraal sa Grade 12 mula sa dalawang track ayon sa pagkakabuo. Batay sa pagsusulit ang dalawang track ay nakakuha ng mababa sa $75 \%$ o fairly satisfactory na inaasahang pasadong marka. Ang lahat ng track ay nakakuha ng did not meet expectation na antas ng kaalaman: ang Academic Track ay nakakuha ng 74.0\%, at ang TLE and TVL Track ay may rating na 69.3\% na rating.

Pinapakita ng resultang ito na ang mga mag-aaral sa senior high school sa Mataas na Paaralan ng Lungsod ng Sorsogon ay hindi sapat ang kaalaman sa pagbuo ng isang pananaliksik. Kulang ang kaalaman nila sa pagtukoy sa iba't ibang bahagi ng pananaliksik, paglalahad ng datos, at metodo sa pangangalap ng mga datos.

Magkakaroon ng suliranin ang mga mag-aaraal sa pagbuo ng pananaliksik sapagkat ang mga kakulangan ng kaalaman ng mag-aaral ay magreresulta sa maling pagbibigay interpretasyon sa datos na nakalap sa pananaliksik na ginagawa at maging ang paraan ng paglalahad nito ay hindi magiging malinaw. Batay nga kay Ordonez, et.al.(2007) ang pananaliksik ay pahayag sa mataas ng lebel ng pagsulat dahil nangangailangan ito ng pangangalap ng mga datos, pag-iimbestiga, pagsusuri, pagbibigay hinuha, at sa pagtatapos ay pagbibigay kongklusyon at rekomendasyon.

Ayon sa kwalitatibong pag-aaral ni Conception (2006), kung ginagamitan ng prosesong pagdulog ang mga sulating nabubuo ng mga mag-aaral ay mas mataas ang kalidad ng mga ito. Napatunayan din na nakatutulong ang prosesong pagdulog sa paglinang ng komprehensyon ng mga mag-aaral. Mas mainam din na isaalang-alang ang kakayahan, istilo, at interes ng mga mag aaral upang mapalago ang komprehensyon nila sa pananaliksik (Conception, 2006).

\subsection{Antas ng kaalaman ayon sa mekaniks ng sulating pananaliksik}

Sa pagtukoy sa antas ng kaalaman ng mga mag-aaral ayon sa mekaniks ng sulating pananaliksik, ay isinaalang-alang ang mga sumusunod na kasanayang pampagkatuto: (1) Maayos na pagsusunod-sunod ang tamang datos sa pagsulat ng isang bibliograpi, at (2) nagagamit ang mga etika ng isang mahusay na mananaliksik, gayundin ang ankop na salita at bantas sa proseso ng pagsulat. Ang mekaniks ng sulating pananaliksik ay 
Pagdalumat sa kaalaman ng mga mag-aaral ng senior high school sa sulating pananaliksik

tumutukoy sa kaalaman ng proseso o paraan ng mag-aaral sa pagsulat ng pananaliksik. Kasama rin ditto ang tamang gamit ng mga salita at pagbabantas.

\section{Talahanayan 1C}

Antas ng kaalaman ng mga mag-aaral sa Grade 12 ng iba't ibang track sa sulating pananaliksik ayon sa mekaniks

\begin{tabular}{lcl}
\hline \multicolumn{1}{c}{ Track } & Rating $(\%)$ & \multicolumn{1}{c}{ Antas ng Kaalaman } \\
\hline Academic Track & 75.5 & Fairly satisfactory \\
TLE and TVL & 69.9 & Did not meet expectation \\
Kabuuang rating & 72.7 & Did not meet expectation \\
\hline
\end{tabular}

Ang talahanayan 1C ay nagpapakita ng resulta ng mga mag-aaral mula sa iba't ibang track matapos ang pagsusulit na nakatuon sa mekaniks ng pananaliksik. Ang Academic Track ay nakakuha ng pasadong rating na $75.5 \%$ o Fairly satisfactory, ang TLE and TVL Track naman ay may $69.9 \%$ na rating o did not meet expectation.

Bagamat nakakuha ng pasadong rating ang Academic Track ang TLE and TVL track ay nasa did not meet expectation pa rin. Sa resultang ito ipinakikita ang kahinaan ng mga mag-aaral sa tamang pagsulat ng tala ng sanggunian, teknikalidad sa pagsulat, at tamang etika na dapat taglayin ng isang manunulat ng pananaliksik.

Mahalagang magkaroon ng sapat na kaalaman ang mag-aaral sa pagsulat ng tala ng sanggunian upang maiwasan ang plahiyo. Binanggit nina Constantino at Zafra, n.d. ang ilan sa mga anyo ng plahiyo sa pananaliksik ito ay maaring tahasang pag-angkin sa gawa ng iba, pagkopya sa ilang bahagi nang may kaunting pagbabago sa ayos ng pangungusap at hindi kinilala ang awtor, at pag-angkin at/o paggagaya sa pamagat ng iba.

Ayon kina Xing at Jin (1989), ang wastong gamit ng mga pananda, talasalitaan, pagbubuo ng kaisipan, retorika at iba pang elemento ang mga kailangan upang magkaroon ng isang komprehensibong pagsulat. Sapagkat ang pagsulat ay isang cognitive na proseso ng pagsasalin ng mga nabuong salita, simbolo at ilustrasyon ng isang bagay o pangyayari upang makabuo ng isang payak na ideya (Bernales et al., 2001). Ayon pa kina Bernales, et al. (2001), ito ay kapwa pisikal at mental na aktiviti o gawain para sa iba't ibang layunin.

\subsection{Makabuluhang pagkakaiba ng antas ng kaalaman ng mga mag-aaral sa iba't ibang track ayon sa mga nabanggit na baryabols}

Sa suliranin ng pananaliksik na ito, nais alamin ng mananaliksik kung mayroon bang makabuluhang pagkakaiba ang kaalaman ng mga mag-aaral ukol sa mga baryabol ng sulating pananaliksik. Upang maanalisa ang mga datos na nakalap ay gumamit ng f-test ang mananaliksik. Ginamit naman ang Scheffe's test upang malaman kung aling track ang naiiba ang resulta.

\section{Talahanayan 2}

Pagkakaiba ng Antas ng kaalaman ng mga mag-aaral sa Grade 12 ng iba't ibang track sa sulating pananaliksik ayon sa nilalaman, pagkakabuo, at mekaniks

\begin{tabular}{lccc}
\multicolumn{1}{c}{ Statistical Bases } & Nilalaman & $\begin{array}{c}\text { Statistical Analyses } \\
\text { Pagkakabuo }\end{array}$ & Mekaniks \\
\hline Level of Significance & 0.05 & 0.05 & 0.05 \\
Degrees of freedom & 14 & 14 & 14 \\
$\mathrm{~F}_{0.05}$ & 2.14 & 2.14 & 2.14 \\
$\mathrm{~F}_{\text {computed }}$ & 7.64 & 7.44 & 6.58 \\
Decision on Ho & Reject & Reject & Reject \\
Conclusion & Significant & Significant & Significant \\
\hline
\end{tabular}

Ang talahanayan 2 ay nagpapakita ng pagkakaiba-iba ng antas ng kaalaman ng mga mag-aaral sa sulating 
pananaliksik ayon the tatlong baryabol gamit ang istadistikang $\mathrm{t}$-test.

Nilalaman. Mula sa Talahanayan 2, makikita na ang computed f-value sa .05 level of confidence ay 7.64 na mas mataas sa f-tabular value na 2.14 kung ang degrees of freedom ay 14. Ang nais ipahiwatig nito ay kailangang i-reject and null hypothesis. Samakatuwid, ang datos na nakuha sa dalawang tracks ayon sa antas ng kaalaman ng mga mag-aaral sa nilalaman ng sulating pananaliksik ay may makabuluhang pagkakaiba. Nais ipahiwatig nito na ang mga tracks ay may iba-ibang antas ng kaalaman sa nilalaman ng sulating pananaliksik mula sa pagpili ng paksa, pagsulat ng pamagat, layunin, paglalahad ng suliranin hanggang sa mga baryabolsna maaring suriin.

Pagkakabuo. Makikita rin sa talahanayan 2 na ang f-computed value sa .05 level of significance ay 7.44. Ang nacompute na f-value ay lagpas sa f-tabular value na 2.14, dahil dito ang null hypothesis ay kailangang i-reject. Ibig sabihin, ang mga tracks ay may iba-ibang antas ng kaalaman ayon sa pagkakabuo ng sulating pananaliksik mula sa mga panimulang impormasyon, mga bahagi ng pananaliksik, pagakakasunod-sunod sa paglalahad ng mga datos at mga ibat-ibang metodo na gagamitin.

Mekaniks. Mula sa talahanayan 2, mapapansin na 6.58 ang nakuhang calculated f-value mula sa mga datos na inanalisa. Ang balyu na ito ay lagpas sa f-tabular value na 2.14 sa .05 level of significance. Dahil sa lagpas ito sa f-tabular, ang null hypothesis ay kailangang i-reject. Sa makatuwid, ang antas ng kaalaman ng mga mag-aaral sa Grade 12 sa GAS at TLE \& TVL Track sa sulalating pananaliksik ayon sa mekaniks ay may makabuluhang pagkakaiba. Ibig sabihin, ang antas ng kaalaman ng mga mag-aaral sa Grade 12 ukol sa mekaniks ng sulating pananaliksik ay nagbabago sa bawat track mula sa paggamit ng mga impormasyong ginagamit sa pagsulat ng mga talaan ng sanggunian, at tamang pagsulat ng mga nakalap na datos o impormasyon sa pananaliksik

Mula sa mga datos na inanalisa, makikitang ang Academic Track ay makabuluhang pagkakaiba sa TLE \& TVL Tracks. Mahihinuha na ayon sa nilalaman, pagkakabuo, at mekaniks ng sulating pananaliksik ay mataas ang nakuhang rating ng Academic track kumpara sa TLE \& TVL Track.

\section{Talahanayan 3}

Pagkakaiba ng Antas ng kaalaman ng mga mag-aaral mula sa GAS at TLE\&TVL Track ayon sa mga nabanggit na Baryabols

\begin{tabular}{cccc}
\hline $\begin{array}{c}\text { Comparison between } \\
\text { strand }\end{array}$ & F Computed Value & F Critical Value & Interpretation \\
\hline GAS vs TVL & 30.16 & 3.95 & Significant \\
\hline
\end{tabular}

Makikita sa talahanayan 3 na ang computed value na 30.16 ay mas mataas sa critical value na 3.16, kaya kailangang i-reject ang null hypothesis. Nangangahulugang mayroong makabuluhang pagkakaiba ang means ng GAS at TLE\&TVL track sa Mataas na Paaralan ng Lungsod ng Sorsogon. Maaaring ipagpalagay na ang kaibahan sa resulta ay dulot ng Alternative Entry Requirement at pagkakaroon ng Special Curriculum Program ng Deped.

Mula sa Deped Order No. 55 s. 2016 o "Policy Guidelines on the National Assessment of Student Learning for the K to 12 Basic Education Program" ay isinalaysay ang mga krayteriya upang makapasok ang mga Junior High School sa Senior High School Tracks/Strands. Nakasaad na mayroong "Alternative Entry Requirements" ang mga mag-aaral ng Junior High School depende sa kung anong track ang kanilang papasukin (DEPED, 2016).

Ang mga pipili ng Academic track ay kailangang gumawa ng sanaysay habang ang sa TLE \& TVL ay kailangan naman ng praktikal na pagsusulit (DEPED, 2016). Mula sa paraan ng pagpasok sa mga Senior High School tracks ay malaking bahagi na ang kaalaman ng mga mag-aaral sa pagsusulat. Sa una pa lang ay inaasahan na ang mga nasa Academic track na may kakayahang pasulat habang ang nasa TLE\&TVL tracks naman ang pokus ay ang paghubog sa kanilang pratikal na kakayahan. Ang pagkakaroon ng Special Curricular Program ng

48 Consortia Academia Publishing (A partner of Network of Professional Researchers and Educators) 
Pagdalumat sa kaalaman ng mga mag-aaral ng senior high school sa sulating pananaliksik

Deped ay isa ring nakakaapekto sa kakayahan ng mga mag-aaral sa pagsulat at pananaliksik.

Batay sa Deped Order no. 46 s. 2012 o ang "Policy Guidelines on the Implementation of the Special Curricular Programs at the Secondary Level", ang mga nasa Special Curricular Programs ay kailangang palitan ang asignaturang TLE ng mga specialized subjects na nakabase sa kanilang ispesipik na programa. Karagdagan pa, sa pagpapatibay ng Deped Order no. 15 s. 2014, ang mga nasa Science, Technology and Engineering (STE) na programa ay may karagdagang asignaturang pananaliksik na maaaring naging dahilan kaya ang mga nasa Academic track ay mas handa sa sulating pananaliksik.

\subsection{Mga salik na nakaaapekto sa kakayahan ng mga mag-aaral sa pagsulat ng pananaliksik ayon sa mga nabanggit na mga Baryabol}

Ang mababang antas ng kaalaman ng mga mag-aaral sa sulating pananaliksik ay dahilan ng iba't ibang mga suliranin. Sa parteng ito ay inilalahad ang mga pangunahing problem na kinaharap ng mga mag-aaral sa Grade 12 na nakaapekto sa kakayahan nilang magsulat ng pananaliksik.

\section{Talahanayan 4}

Mga salik na nakaaapekto sa kakayahan ng mga mag-aaral sa pagsulat ng pananaliksik

\begin{tabular}{|c|c|c|}
\hline \multirow{2}{*}{ Mga Salik } & \multicolumn{2}{|c|}{ Frequency and rank } \\
\hline & Academic & TVL/TLE \\
\hline 1. Nahihirapang bumuo ng pamagat para sa pananaliksik & $20(1)$ & $11(1)$ \\
\hline 2. Limitado ang mga sanggunian para sa ninanais na paksa & $13(4.5)$ & $9(3.5)$ \\
\hline $\begin{array}{l}\text { 3. Wala pang mga nakalipas na pag-aaral hinggil sa paksang } \\
\text { napili na magsisilbing batayan sa kasalukuyang pananaliksik }\end{array}$ & $9(9.5)$ & $7(8.5)$ \\
\hline $\begin{array}{l}\text { 4. Kung pangkatan ang ginagawang pananaliksik, nagiging } \\
\text { balakid ang ibang kapangkat sapagkat hindi gaanong } \\
\text { nakikisangkot }\end{array}$ & $16(2)$ & $10(2)$ \\
\hline $\begin{array}{l}\text { 5. Hindi sapat ang panahong nakalaan upang matapos ang } \\
\text { isang pananaliksik }\end{array}$ & $9(9.5)$ & $8(5.5)$ \\
\hline $\begin{array}{l}\text { 6. Hindi sapat ang kakayahan sa pagsasagawa ng isang } \\
\text { pananaliksik }\end{array}$ & $13(4.5)$ & $8(5.5)$ \\
\hline 7. Kulang ang kaalaman sa paksang pinag-aaralan & $13(4.5)$ & $8(5.5)$ \\
\hline $\begin{array}{l}\text { 8. Limitado ang budyet ng isang mag-aaral para } \\
\text { makapagsagawa ng isang pananaliksik }\end{array}$ & $13(4.5)$ & $8(5.5)$ \\
\hline $\begin{array}{l}\text { 9. Hindi gaanong malinaw ang konsepto ng mag-aaral sa } \\
\text { pagsasagawa ng isang pananaliksik }\end{array}$ & $13(4.5)$ & $7(8.5)$ \\
\hline $\begin{array}{l}\text { 10. Kulang ang mga nakalap na kaugnay na literature at } \\
\text { kaugnay na pag-aaral sa paksang napiling saliksikin }\end{array}$ & $15(3)$ & $9(3.5)$ \\
\hline
\end{tabular}

Makikita sa talahanayan 4 ang mga salik na nakaaapekto sa kakayahan ng mga mag-aaral sa pagsulat ng pananaliksik. Mula sa 10 salik ipinatukoy sa mga kalahok kung anong salik ang kanilang naranasan sa pagsulat ng sulating pananaliksik.

Makikita sa talahanayan 4 na ang Academic Track at TLE/TVL Track ay nagkakaisa na ang pagbuo ng pamagat ang nangungunang salik na nakaaapekto sa pagsulat ng pananaliksik. Batay sa naging resulta mula sa 40 na kalahok mula sa Academic Track ang pumili sa salik na ito 20 mula naman sa TLE and TVL Track ay 11. Isa ito sa pinatunayan nina Anson at Smith (2004) sa kanilang pag-aaral. Ayon sa kanila ang mga mag-aaral ay nahihirapang magdesisyon kung ano ba ang mga paksa ng pananaliksik na magagawa at iilan lang ang may malinaw na pokus sa kung ano ang dapat nilang gawin sa saliksik.

Nagkaisa naman ang dalawang track na ang nasa ikalawang ranggo ay ang salik na kung pangkatan ang ginagawang pananaliksik, nagiging balakid ang ibang kapangkat sapagkat hindi gaanong nakikisangkot. Mayroong 15 na mag-aaral mula sa Academic Track ang nagbigay ng puntos dito, 10 naman sa TVL/TLE Track. Isa sa mga balakid sa pagsasagawa ng anumang gawain ay ang kakulangan ng pakikiisa ng mga taong kasangkot, 
sa halip na maging magaan ang gawain ay mas nagiging kumplekado pa ito. Ayon kay Bernales et.al.(2009)may mga katangiang kailangang taglayin ang mananaliksik ito ay ang sumusunod; una,dapat na maging masipag ang mananaliksik sapagkat ang pangangalap na datos ay hindi madaliang gawain. Ikalawa, ang pagiging matiyaga dahil ang pananaliksik ay may mga prosesong dapat na sundin hindi ito maisasakatuparan ng madalian lamang. Ikatlo ang pagiging mapanuri, dapat ay maalam ang isang mananaliksik kung ano ang mga akma at makabuluhang datos na maaring gamitin sa kanyang pag-aaal. At dapat ay may kakayahang timbangin at bigyan ng sapat na pangangatwiran ang mga impormasyong nakalap. Kung ang mga katangiang ito ay tataglayin ng mga mag-aaral sa kanilang pagsulat ng pananaliksik magiging magaan ang kanilang gawain at hindi magiging sagabal ang mga kapangkat.

Sinasang-ayunan din ng dalawang na Tracks na isa ang salik na kulang ang mga nakalap na kaugnay na literatura at kaugnay na pag-aaral sa paksang napiling saliksikin sa mga salik na nakaaapekto sa kakayahan ng mag-aaral sa pagsulat ng pananaliksik. Napakahalaga ng mga kaugnay na literature at pag-aaral sa isang pananaliksik sapagkat ito ang nagsisilbing mga patunay sa nais saliksikin at dito din nakukuha ang mga kasagutan sa pag-aaral na isinasagawa maraming mga maaaring mapagkunan ng mga impormasyon nariyan ang mga aklat, journal, artikulo, at internet. Kung ang isang mananaliksik ay magiging matiyaga lamang sa pangagangalap ng mga impormasyon hindi magiging balakid ang kakulangan ng kaugnay na literature at pag-aaral sa pananaliksik. Ayon kay Sauco (1998), ang pananaliksik ay isang pamamaraang sistematiko, pormal at masaklaw na pagsasagawa ng pagsusuring lohiko at wasto sa pamamagitan ng matiyaga at hindi apurahang pagkuha ng mga datos.

Ang mga salik na ito ay nagiging balakid sa mga mag-aaral upang makapagsulat ng sulating pananaliksik. Subalit, kung titingnang mabuti ang mga salik na nabanggit ay madali lamang masolusyunan kung mayroong disiplina ang mga mag-aaral at ibibigay nila ang kanilang pokus sa pagsulat.

\section{Kongklusyon at Rekomendasyon}

Ang antas ng kaalaman ng mga mag-aaral sa grade 12 sa nilalaman, pagkakabuo, at mekaniks ay nasa below 75 o did not meet expectation. Tanging ang Academic track lamang ang may pasadong rating sa mekniks, nagtamo ng $75.5 \%$ o fairly satisfactory. Ang antas ng kaalaman ng mga mag-aaral mula sa dalawang track ay may makabuluhang pagkakaiba sa nilaman, pagkakabuo, at mekaniks. Mula sa mga datos na nakalap at inanalisa ang Academic Track ang nakakuha ng mas mataas na rating kumpara sa TLE \& TVL Track. Nagkakaisa ang dalawang track na ang pagbuo ng pamagat para sa pananaliksik ang salik na nakaaapekto sa kakayahang sumulat ng pananaliksik.

Batay sa natuklasan at kongklusyon ang inirerekomenda ay ang sumusunod. Magbigay ng mas maraming gawaing pagsasanay sa mga mag-aaral upang malinang ang kanilang pagkatuto sa nilalaman, pagkakabuo, at mekaniks ng pananaliksik. Gamitin ang localization sa pagtuturo ng asignaturang "Pagbabasa at Pagsusuri ng Iba't ibang Teksto Tungo sa Pananaliksik”. Mas bigyang pansin ang paglinang sa kakayahan ng mga mag-aaral sa paghahanap ng solusyon sa mga maaaring pag-aralang mga problema. At sanayin ang mga mag-aaral sa pagsusuri ng mga kaugnay na literature at pag-aaral na maaaring magamit sa pananaliksik.

\section{Talasanggunian}

Almario, V. S. et.al.(2016). Introduksyon sa Saliksik. San Miguel, Maynila. Komisyon sa Wikang Filipino.

Alvesson, M., \& Sandberg, J. (2013). Constructing research questions: doing interesting research. London: Sage, Thousand Oaks, CA: Sage Publications.

Anson, R., \& Smith, K. (2004). Undergraduate research projects and dissertations: issues of Topic Selection, Access and Data Collection Amongst Tourism Management Students. from http://www.hist.ltsn.ac.uk/johiste

Badayos, P. B. (2005). Metodolohiya sa Pagtuturo ng Wika. Makati City: Grandwater Publications. 
Pagdalumat sa kaalaman ng mga mag-aaral ng senior high school sa sulating pananaliksik

Balona, J. B. (2018.) Writing profeceincy and needs of the grade 9 students in Bulan Cluster, Sorsogon State College. School of Graduate Studies.

Bernales, R. A. et.al. (2008). Kritikal na Pagbasa at Lohikal na Pagsulat sa Pananaliksik. Valenzuela City: Mutya Publishing House.

Bernales, R. A. et.al. (2009). Mabisang Komunikasyon sa Wikang Pang-akamemiko. Valenzuela City: Mutya Publishing House.

CIIT Education PH. 2017. Retrieved November 22, 2021

https://www.ciit.edu.ph/senior-high-school-track/

Conception, Ofelia E. 2008. Diksyunaryong Filipino-Filipino. Harmony Publishing House.

Constantino, P. C. at Zafra, G. S. (1997). Kasanayan sa Komunikasyon II. Quezon City. University of the Philippines Open University.

Department of Education. 2012. Retrieved November 22, 2021 from

https://www.deped.gov.ph/wp-content/uploads/2012/06/DO_s2012 46.pdf

Department of Education. 2014. Retrieved November 22, 2021 from https://www.deped.gov.ph/wp-content/uploads/2014/03/DO_s2014_015.pdf

Department of Education. 2015. Retrieved November 22, 2021 from https://www.deped.gov.ph/wp-content/uploads/2015/04/DO_s2015_08.pdf

Department of Education. 2016. Retrieved November 22, 2021 from https://www.deped.gov.ph/wp-content/uploads/2016/06/DO_s2016 55-3.pdf

Department of education. 2019. Retrieved November 22, 2021 from https://www.deped.gov.ph/wp-content/uploads/2019/01/SHS-Core Pagbasa-at-Pagsusuri-ng-I bat-Ibang-Teksto-Tungo-sa-Pananaliksik-CG.pdf

Lecter, J. K. et.al. (2011). Instruksyong Modyular sa Pananaliksik. Lungsod ng Mandaluyong: Anvil Publishing, Inc. 
Ojos, M. K. H., Deyto, N. H., \& Janer, S. S.

52 Consortia Academia Publishing (A partner of Network of Professional Researchers and Educators) 